\title{
Exploring Potential Neurobiological Parallels of Restrictive Behaviour: Anorexia Nervosa and Chew and Spit (CHSP)
}

Phillip Aouad ${ }^{1,{ }^{*},}$ Nerissa Soh ${ }^{2,+}$, Kristin Stedal $^{3,+}$

1. InsideOut Institute, The Boden Institute - Charles Perkins Centre (D17), University of Sydney, Sydney 2050 Australia; E-Mail: phillip.aouad@sydney.edu.au

2. Honorary Associate, Northern Clinical School, Faculty of Medicine and Health, University of Sydney, Sydney, Australia; E-Mail: nerissa.soh@sydney.edu.au

3. Regional Department for Eating Disorders, Division of Mental Health and Addiction, Oslo University Hospital, Oslo, Norway; E-Mail: kristin.stedal@ous-hf.no

+ These authors contributed equally to this work.

* Correspondence: Phillip Aouad; E-Mail: phillip.aouad@sydney.edu.au

Academic Editor: Sarah Maguire

Special Issue: Neurobiological Underpinnings of Anorexia Nervosa

OBM Neurobiology

2020, volume 4, issue 3

doi:10.21926/obm.neurobiol.2003070
Received: May 05, 2020

Accepted: August 16, 2020

Published: August 18, 2020

\begin{abstract}
Consensus for where along the eating disorder spectrum the pathological eating behaviour of Chew and Spit (CHSP) falls has been a point of contention in academic literature. Using the case of Mary ${ }^{1}$, a 30 year old female from Australia who has engaged in excessive restrictive behaviour, was diagnosed with anorexia nervosa (AN) and has been engaging in Chew and Spit (CHSP) behaviour for over 10 years, the current paper examines the similarities from a neurobiological perspective between restrictive behaviours (akin with AN) and CHSP. In order to better understand the similarities between restrictive and CHSP
\end{abstract}

\footnotetext{
${ }^{1}$ Name has been changed to protect privacy
}

(C) 2020 by the author. This is an open access article distributed under the conditions of the Creative Commons by Attribution License, which permits unrestricted use, distribution, and reproduction in any medium or format, provided the original work is correctly cited. 
behaviours and to begin gaining insight into potential approaches to dealing with CHSP as a primary symptom of an eating disorder, this paper aims to draw neurobiological parallels between CHSP and restrictive behaviours. Based on the behaviours outlined in the case provided, the paper broadly examines the anatomy of particular brain regions, structures and pathways before highlighting that similar reward processing and inhibition pathways could be dysregulated in both restrictive behaviours, seen in AN, and CHSP. This short communication paper draws a neurobiological similarities between restrictive behaviours akin to AN and CHSP, and offers points of contemplation, discussion, and provide a logical stepping-stone in order to examine CHSP more closely. The clinical significance of current neurobiological approaches to the treatment of AN are also considered in relation to CHSP. Future studies may focus on studying such neurobiological interventions as well as conducting functional brain imaging studies to better understand if the hypothesised brain regions and pathways are implicated in CHSP.

\section{Keywords}

Chew and spit; CHSP; anorexia nervosa; bulimia nervosa; eating disorders; OSFED; feeding and eating disorder; neurobiology; dopaminergic; neural pathways

\section{Case Overview}

Mary (pseudonym used to maintain anonymity), a 30 year old female from Australia, has been diagnosed with multiple eating disorders over the course of her life, with the first diagnosis being Eating Disorder Not Otherwise Specified (EDNOS) at the age of 7 years old (further discussion indicates that the diagnosis was akin to the current DSM-5 Avoidant/ Restrictive Food Intake Disorder (ARFID) [1]. In her teenage years, Mary was hospitalised due to Anorexia Nervosa (AN) with a $\mathrm{BMI}<16$, which led to a myriad of health complications. During recovery Mary notes she began to engage in chewing and spitting (CHSP) out food, which she reported helping her feel as though she is "continuing restricting", however, she did not consider this a total relapse. She reported that she had been engaging consistently in CHSP for over 10 years, and that the behaviour not only feels addictive, but soothing or calming as well, and that she initially derived pleasure followed by shame/guilt when engaging in the behaviour. Mary has attempted many treatments (with some repeat treatments) over the years including family-based therapy, the Maudsley program, and cognitive behaviour therapy (CBT/CBT-E). While Mary has recovered from AN, CHSP still persists as her primary disordered eating behaviour and she now considers it her 'new normal'. Ultimately, Mary would like to stop engaging in CHSP altogether.

\section{Introduction}

Anorexia Nervosa (AN) is characterised by persistent caloric restriction, often perpetuated by intense underlying fear of gaining weight [2]. Individuals with AN often use excessive methods in order to control or maintain a low body weight related to their expected growth rate, height, sex, and age [2]. 
Chew and Spit (CHSP) is a behaviour characterised by chewing food and discarding the contents of the mouth prior to swallowing [3]. In individuals with eating disorders, this is often done in order to restrict the intake of calories or nutrients from the food itself, while still enjoying an otherwise subjectively 'forbidden 'or 'unsafe' food [3].

CHSP, as a symptom of Eating Disorders Not Otherwise Specified (ENDOS), was listed in the American Psychiatric Association Diagnostic and Statistical Manual (DSM) up until version 4 [1]. However, since 2013, it has been formally removed as a listed clinical symptom from any of the featured eating disorders in the DSM-5 [1], potentially due to lack of available evidence on the prevalence and ramifications of engaging in CHSP. Its removal from diagnostic manuals may subsequently remove the behaviour from clinical awareness, which means the behaviour goes unscreened and unaddressed during the course of treatment for other eating disorders.

Case studies like the one described above highlight that CHSP behaviours indeed still exist despite decreasingly being screened for since the removal of CHSP from the DSM-5, even during recovery for a diagnosed eating disorder. In addition, recent point-prevalence studies have indicated that CHSP could be present in $0.4 \%$ of adults [4] and upwards of $12 \%$ in adolescents [5]. These studies also highlight that, compared to the general population, individuals who engage in CHSP may experience: lower mental and physical health related quality of life, increased negative emotions and intrusive thoughts, have more health concerns related to CHSP - including subsequent weight gain, and have dysregulated or impaired psychosocial wellbeing $[5,6]$. Despite these severe negative associations with engaging in CHSP, there is a paucity in academic and clinical understanding about CHSP and its underlying pathology [3]. On the other hand, for restrictive-type disorders, such as $\mathrm{AN}$, there is currently a growing body of evidence suggesting that neurobiological factors which influence behaviour and individual temperament are involved in the maintenance of restrictive behaviours [7-9]. Restrictive-type eating disorders, such as AN, are often characterised by high levels of anxiety, perfectionistic tendencies, dysregulated interoception (sensitivity to internal cues) and response to reward, and cognitive inadaptability related to the physiological differences in the brain, primarily the insula and fronto-striatal circuitry [10-12]. It could be hypothesised that some of these brain circuits are also involved in the maintenance of CHSP behaviours. The restrictive behaviours seen in AN are often explained as being caused by unbalanced reward processing, inhibition control and overall dysregulated eating behaviours. Due to the restrictive nature of CHSP behaviours, it is plausible that the same - or similar - neurocognitive processes are involved in CHSP.

The current study aims to provide an initial examination of CHSP along the spectrum of eating disorder behaviours, with the focus being on the neurobiological similarities that may be present between CHSP and the type of restrictive behaviour seen in AN. The case overview of Mary (described above) serves to: 1 . highlight some of the behavioural similarities between restricting behaviours seen in AN and the behaviour of CHSP; 2. highlight that CHSP (as a behaviour) may fall along the spectrum of eating disorders symptoms, and 3. CHSP may be transient but can also present as a primary eating disorder symptom. While this paper aims to highlight potential neurobiological similarities between the restrictive characteristics of CHSP and AN, it should be noted from the outset that the paper does not aim to argue that CHSP is a stand-alone disorder, and it also acknowledges that limitations exist in comparing CHSP (a behaviour) to AN (a syndrome/ diagnosable eating disorder). 
Mary is a patient who has been diagnosed with AN, where CHSP remains a primary symptom of her diagnosis for over a decade. Her case illustrates the need for more insight into the neurobiological underpinnings between CHSP and restrictive-type eating disorders as well as highlighting the need for treatments specifically targeted at CHSP. To date, no studies have investigated the neurobiology of CHSP. Given the alignment of key characteristics between CHSP and $A N$ in regard to restrictive behaviours, exploring the existing evidence-base of the neurobiology of AN could aid in developing hypotheses about the neurobiological underpinnings of CHSP. This could provide a framework for future studies in order to better understand the similarities between restriction and CHSP, and to begin gaining insight into potential approaches to dealing with CHSP as a primary symptom of an eating disorder. Ultimately, this paper aims to draw neurobiological parallels between the similarities of CHSP and restrictive-type AN in order to provide a foundation and framework for future studies.

\section{Anatomy and Neurobiological Overview}

In the past decade, an increasing amount of research has focussed on the gut-brain (hypothalamic) link, which various studies have both directly and indirectly attributed to the regulation of metabolism, emotional regulation, and by extension eating behaviours [7, 13, 14]. Broadly, the corticolimbic system is believed to influence the pathophysiology of eating disorders, with three specific networks in the corticolimbic system being responsible for the regulation of eating disorders [7].

\section{First Network}

The first network includes the insula and anterior cingulate and insula and frontal operculum, which are believed to be involved in the ability to decipher the importance of feeding and in the processing of sensory information related to food, respectively [7].

\section{Second Network}

The second network includes the nucleus accumbens, putamen, caudate, orbitofrontal cortex and amygdala, and is involved in maintaining one's aspect of reward and motivation to eat, which may contribute to an individual's approach or avoidance behaviour [7].

Third Network

The third network is known as the dopaminergic pathway, which includes the dorsal caudate and dorsal anterior cingulate, lateral prefrontal cortex, and parietal cortex, and is responsible for controlling food consumption in the context of short and long term outcomes, such as weight loss or gain [7].

The three corticolimbic networks, in composite, are involved in 'weighing up' the value and reward of eating the food vs. the consequence of eating, swallowing, or ingesting it. Additionally, findings suggest that difference in neurocircuitry may also be linked to temperament traits, like anxiety or harm-avoidance, which may underlie eating pathology development [7]. Further evidence indicates the disequilibrium between reward and inhibition in those with eating disorders. For example, an individual with AN will undertake severe food restriction despite being hungry, severely malnourished, and experiencing a myriad of medical complications related to the restriction of energy intake [7].

Similarly, it can be hypothesised that individuals who engage in CHSP are also overriding homeostatic signals and reflexes (such as the palatal, or swallowing, reflex). Specifically, 
individuals who engage in CHSP, at times appear unable to determine the importance of feeding in a consistent manner, especially if CHSP is a primary symptom of an individual with an eating disorder, and episodes occur at a higher frequency than normal eating patterns. Similar to other restrictive behaviours, individuals who engage in CHSP may use the behaviour to ultimately avoid dealing with negative emotions, which arise from the fear of eating and the fear of gaining weight [6]. Finally, the ability for an individual with CHSP to appropriately distinguish between reward and punishment when it comes to food appears to result in an internal dilemma -specifically, between being able to taste the food but without ingesting it.

\section{Reward Processing}

The brain has different responses when it is anticipating food, to when food is being consumed. However, the difference in brains of individuals with an eating disorder, is that brains change how anticipation and processing of food as a reward may drive their approach to eating [15]. In brains of individuals without an eating disorder, the ventral-striatum and dorsal-caudate have an increased response to reward versus punishment [7]. However, individuals with eating disorders have been demonstrated to show dysregulated reward valuation [7]. Dysregulation of dopamine levels, specifically dopaminergic pathways, have been implicated as key mechanisms involved in the perception of food being seen as reward or punishment in some individuals with different types of eating disorders [16-18]. For example, in individuals with Binge Eating Disorder (BED) and Bulimia Nervosa (BN) there appears to be a decreased response in the insula and ventral striatum brain regions, especially when anticipating food as a reward; however, an increase in response in the same regions is seen when the food is consumed [7].

This is in contrast to individuals with AN (restricting), where studies have shown an increase in both anxiousness and anticipation for sweet-tasting food [7]. The neural responses associated with increased anxiousness and anticipation is seen in the insula, striatum, \& prefrontal cortex; this contrasts with a decreased response in the insula \& striatum to the actual consumption of the food [7]. Thus, in individuals with AN there appears to be conflicting neurobiological responses when anticipating food and consuming the food. A similar conflict can be seen in the behaviour of individuals with CHSP. They often crave sweet food [3], but are unable to allow themselves the 'reward' of consuming (swallowing) it and end up expelling the contents of the mouth. While the behaviour may be more overt in individuals with CHSP compared to individuals with AN, it stands to reason that a similar (if not the same) type of neurobiological process involving reward and punishment is occurring.

\section{Self-Control (Inhibition)}

Individuals with eating disorders have difficulty regulating their behaviour around food, control of weight and shape, or exercise regimes. Reduced inhibition may relate to disequilibria of the frontostriatal circuits, which is responsible for behaviour regulation [7]. Specifically, inefficient or insufficient activation of the frontostriatal circuits may result in difficulty in limiting/stopping eating and may even extend beyond the eating disorder to other behaviours, such as self-harm, kleptomania, or substance misuse [7]. In contrast, an increase in cognitive control in AN may explain severe restricting, even when weight has become dangerously low [7]. This may be due to an increase in activity in the dorsolateral cognitive circuitry, which is associated with inhibition 
regulation and decision making [7]. Moreover, inhibition regulation may also be stymied due to increased activity in behavioural control brain regions being more active during delayed gratification. Similarly, in individuals with CHSP, inhibition and decision-making processes may be adversely impacted leading to on-going chewing and spitting of food. Additionally, individuals who engage in binge-type CHSP episodes may have increased (overactive) inhibitory control but reduced (underactive) reward circuitry, as is often seen in individuals with $A N[6,7]$.

\section{Future Directions}

Overall, the parallels drawn in the current paper serve as a point of contemplation and discussion, and provide a logical stepping stone in order to further study and better understand the neurobiological underpinnings of CHSP. In AN, the implication of neurobiological research in the field of eating disorders has led to treatments focusing on the potential of neuromodulation, such as Transcranial Magnetic Stimulation (TMS) [19, 20], trans-Cranial Direct Current Stimulation (tDCS) [19], and deep brain stimulation (DBS) [21]. In addition, psychotherapeutic treatments which target the biological basis for the maintenance of maladaptive, including eating pathology and AN-centric behaviours [22] have also been developed as a result of the increased knowledge of neurobiological functioning in eating disorders. Future studies should continue to examine the parallels (both neurobiological and otherwise) between CHSP and other eating disorders, continuing to investigate modes of treatment that may help alleviate CHSP. By understanding the parallels between CHSP and other eating disorders, such as in Mary's case above, and CHSP's relation to $A N$, treatments may be better tailored to reduce and ultimately stop maintaining behaviours such as extreme restriction and CHSP.

\section{Conclusion}

The severe impact of CHSP on psychological wellbeing highlights the need for increased knowledge about this behaviour. There is currently no consensus as to where along the ED spectrum CHSP currently falls; but by drawing neurobiological parallels between CHSP and other eating disorders, knowledge can be gained to better understand how to target CHSP as a potential symptom of eating disorders. Future research may focus on brain imaging of individuals who engage in CHSP as a primary symptom and compare the results with individuals who have been diagnosed with AN. Such imaging studies would greatly benefit academic and clinical understanding of the neurobiological underpinnings of CHSP.

\section{Author Contributions}

PA was responsible for conceptualisation, development, drafting of the initial draft, and editing of subsequent drafts. KS and NS were responsible for development, editing subsequent drafts, and consultation.

\section{Funding}

The current study is not subject to any funding scheme. 


\section{Competing Interests}

The authors have declared that no competing interests exist.

\section{References}

1. American Psychiatric Association. Diagnostic and statistical manual of mental disorders (DSM5). 5th ed. Washington, DC: American Psychiatric Association; 2013.

2. Mancuso SG, Newton JR, Bosanac P, Rossell SL, Nesci JB, Castle DJ. Classification of eating disorders: Comparison of relative prevalence rates using DSM-IV and DSM-5 criteria. $\mathrm{Br} J$ Psychiatry. 2015; 206: 519-520.

3. Aouad P, Hay P, Soh N, Touyz S. Chew and spit (CHSP): A systematic review. J Eat Disord. 2016; 4: 23.

4. Aouad P, Hay P, Soh N, Touyz S. Prevalence of chew and spit and its relation to other features of disordered eating in a community sample. Int J Eat Disord. 2018; 51: 968-972.

5. Aouad P, Hay P, Soh N, Touyz S, Mannan H, Mitchison D. Chew and spit (CHSP) in a large adolescent sample: Prevalence, impact on health-related quality of life, and relation to other disordered eating features. Eat Disord. 2019; 26: 1-14.

6. Aouad P, Morad A, Hay P, Soh N, Touyz S, Rhodes P. Chew and spit (CHSP): An interpretative phenomenological analysis (IPA). Eat Behav. 2020; 37: 101388.

7. Ely A, Berner L, Wierenga C, Kaye W. Neurobiology of eating disorders: Clinical implications. Psychiatr Times. 2016; 33: 1-6.

8. Hill L, Peck SK, Wierenga CE, Kaye WH. Applying neurobiology to the treatment of adults with anorexia nervosa. J Eat Disord. 2016; 4: 31.

9. Wierenga CE, Hill L, Knatz Peck S, McCray J, Greathouse L, Peterson D, et al. The acceptability, feasibility, and possible benefits of a neurobiologically-informed 5-day multifamily treatment for adults with anorexia nervosa. Int J Eat Disord. 2018; 51: 863-869.

10. Klump KL, Bulik CM, Pollice C, Halmi KA, Fichter MM, Berrettini WH, et al. Temperament and character in women with anorexia nervosa. J Nerv Ment Dis. 2000; 188: 559-567.

11. Lilenfeld LR. Personality and temperament. Behavioral neurobiology of eating disorders. Berlin: Springer; 2010. p. 3-16.

12. Berner LA, Simmons AN, Wierenga CE, Bischoff-Grethe A, Paulus MP, Bailer UF, et al. Altered interoceptive activation before, during, and after aversive breathing load in women remitted from anorexia nervosa. Psychol Med. 2018; 48: 142-154.

13. Phillipou A, Rossell SL, Castle DJ. The neurobiology of anorexia nervosa: A systematic review. Aus N Z J Psychiatry. 2014; 48: 128-152.

14. Simon JJ, Stopyra MA, Friederich HC. Neural processing of disorder-related stimuli in patients with anorexia nervosa: A narrative review of brain imaging studies. J Clin Med. 2019; 8: 1047.

15. Stice E, Spoor S, Bohon C, Veldhuizen MG, Small DM. Relation of reward from food intake and anticipated food intake to obesity: A functional magnetic resonance imaging study. J Abnorm Psychol. 2008; 117: 924.

16. Casper RC. The 'drive for activity'and "restlessness" in anorexia nervosa: Potential pathways. J Affect Disord. 2006; 92: 99-107. 
17. Kontis D, Theochari E. Dopamine in anorexia nervosa: A systematic review. Behav Pharmacol. 2012; 23: 496-515.

18. Frank GK. Altered brain reward circuits in eating disorders: Chicken or egg? Curr Psychiatry Rep. 2013; 15: 396.

19. Wonderlich SA, Bulik CM, Schmidt U, Steiger H, Hoek HW. Severe and enduring anorexia nervosa: Update and observations about the current clinical reality. Int J Eat Disord. 2020; 53: 1303-1312.

20. Zis $P$, Shafique F, Hadjivassiliou M, Blackburn D, Venneri A, Iliodromiti S, et al. Safety, tolerability, and nocebo phenomena during transcranial magnetic stimulation: A systematic review and meta-analysis of placebo-controlled clinical trials. Neuromodulation. 2020; 23: 291-300.

21. Liu W, Zhan S, Li D, Lin Z, Zhang C, Wang T, et al. Deep brain stimulation of the nucleus accumbens for treatment-refractory anorexia nervosa: A long-term follow-up study. Brain Stimul. 2020; 13: 643-649.

22. Kaye WH, Wierenga CE, Knatz S, Liang J, Boutelle K, Hill L, et al. Temperament-based treatment for anorexia nervosa. Eur Eat Disord Rev. 2015; 23: 12-18.

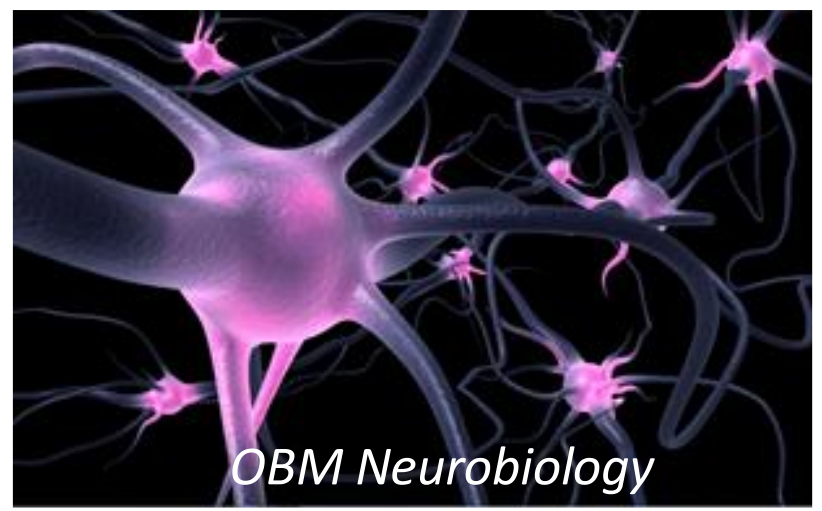

Enjoy OBM Neurobiology by:

1. Submitting a manuscript

2. Joining volunteer reviewer bank

3. Joining Editorial Board

4. Guest editing a special issue

For more details, please visit:

http://www.lidsen.com/journals/neurobiology 